\title{
Superradiant Cascade in a Seeded Free-Electron Laser
}

\author{
L. Giannessi, ${ }^{1, *}$ M. Bellaveglia, ${ }^{2}$ E. Chiadroni, ${ }^{2}$ A. Cianchi, ${ }^{6}$ M. E. Couprie,${ }^{7}$ M. Del Franco, ${ }^{1}$ \\ G. Di Pirro, ${ }^{2}$ M. Ferrario, ${ }^{2}$ G. Gatti, ${ }^{2}$ M. Labat, ${ }^{1,7}$ G. Marcus, ${ }^{8}$ A. Mostacci, ${ }^{4}$ A. Petralia, ${ }^{1}$ \\ V. Petrillo, ${ }^{3}$ M. Quattromini, ${ }^{1}$ J. V. Rau, ${ }^{5}$ S. Spampinati, ${ }^{9}$ and V. Surrenti ${ }^{1}$ \\ ${ }^{1}$ ENEA C.R. Frascati, Via E. Fermi, 4500044 Frascati, Roma, Italy \\ ${ }^{2}$ INFN-LNF, Via E. Fermi, 4000044 Frascati, Roma, Italy \\ ${ }^{3}$ Università degli Studi di Milano and INFN-Mi, Via Celoria, 1620133 Milano, Italy \\ ${ }^{4}$ Università La Sapienza, Piazzale Aldo Moro, 100185 Roma, Italy \\ ${ }^{5}$ ISM-CNR Via del Fosso del Cavaliere, 10000133 Roma, Italy \\ ${ }^{6}$ INFN-Roma Tor Vergata and Università di Roma Tor Vergata, Via della Ricerca Scientifica, 1-00133 Rome, Italy \\ ${ }^{7}$ SOLEIL, L'Orme des Merisiers Saint-Aubin, BP 4891192 GIF-sur-Yvette, Cedex, France \\ ${ }^{8}$ Department of Physics and Astronomy, Particle Beam Physics Laboratory, UCLA, Los Angeles, California 90095, USA \\ ${ }^{9}$ Sincrotrone Trieste S.C.p.A., Area Science Park, S.S. 14 Km 163.5, I-34149 Trieste, Italy
}

(Received 27 September 2012; published 23 January 2013)

\begin{abstract}
We report measurements demonstrating the concept of the free-electron laser (FEL) superradiant cascade. Radiation $\left(\lambda_{\mathrm{rad}}=200 \mathrm{~nm}\right)$ at the second harmonic of a short, intense seed laser pulse $\left(\lambda_{\text {seed }}=400 \mathrm{~nm}\right)$ was generated by the cascaded FEL scheme at the transition between the modulator and radiator undulator sections. The superradiance of the ultrashort pulse is confirmed by detailed measurements of the resulting spectral structure, the intensity level of the produced harmonics, and the trend of the energy growth along the undulator. These results are compared to numerical particle simulations using the FEL code GENESIS 1.3 and show a satisfactory agreement.
\end{abstract}

DOI: 10.1103/PhysRevLett.110.044801

PACS numbers: 41.60.Cr, 41.50.+h, 42.55.Vc

Fourth generation light sources open the way towards the exploration of molecular and atomic phenomena, yielding unprecedented benefits to a wide range of scientific disciplines [1-6]. Free-electron lasers (FELs), operating in self-amplified spontaneous emission (SASE) mode, have demonstrated the capability of reaching the subnanometer wavelength range at the femtosecond time scale $[7,8]$. The multielectron dynamics in atoms and molecules involved in chemical transformation processes evolves on a femtoto attosecond time domain. Thus, ultrashort pulses in the XUV spectral range are the potential tool to control and map the collective electronic and nuclei rearrangements. In a SASE FEL the generation of radiation is due to the passage of a relativistic electron beam through the periodic magnetic field of an undulator with maximum field amplitude $B_{u}$ and period $\lambda_{u}$, inducing emission at a resonant wavelength $\lambda_{\text {rad }}=\lambda_{u}\left(1+K^{2} / 2\right) / 2 \gamma^{2}$ (linear undulator) and its higher order harmonics, where $\gamma$ is the Lorentz factor of the electrons and $K=e B_{u} \lambda_{u} /\left(2 \pi m_{e} c\right)$ the deflection parameter of the undulator. At the onset of saturation, the electrons emit coherently [9] over a characteristic frequency bandwidth $\Delta \omega / \omega \sim \rho$, where $\rho$ is the Pierce parameter $[10,11]$. Considering that typical values of the Pierce parameter range from $10^{-3}$ to $10^{-4}$, the associated FWHM pulse length at the Fourier limit is $\delta \tau=2 \sqrt{2 \ln (2)} L_{c} / c$, where $L_{c}=\lambda_{\text {rad }} / 4 \pi \rho$ is known as cooperation length and, at $\lambda_{\text {rad }}=1 \mathrm{~nm}$, assumes values in the range $\delta \tau_{c} \sim 0.6-6 \mathrm{fs}$. When the electron bunch is longer than $L_{c}$, several independent processes of SASE amplification can occur, leading to a pulse structure far from the Fourier limit and composed of a number of independent spikes [12]. A selective amplification of only one of these spikes has been demonstrated by shaping the electron beam phase space, in order to enable the field growth only in a limited portion of the bunch $[13,14]$. A single mode may also be generated by seeding the FEL amplifier with an external source. Seeding with a pulse shorter than $\delta \tau_{c}$ results in a spike of rms duration given by the cooperation length, at the onset of saturation when $P_{\text {sat }} \approx \rho P_{\text {beam }}\left(P_{\text {beam }}\right.$ is the electron beam power). Pulses shorter than the cooperation length may still be obtained at intensities higher than the saturation level, when the FEL emits in the superradiant regime [15]. In superradiance, the radiation pulse length $\delta \tau_{\mathrm{sr}}$ is a function of the FEL peak power, decreases as $\delta \tau_{\mathrm{sr}} \propto z^{-1 / 2}$ ( $z$ is the distance along the undulator), and can become significantly shorter than that of the input seed laser [16]. The peak power $P_{\text {sr }}$ increases as $P_{\mathrm{sr}} \propto z^{2}$ and the pulse energy $E_{\mathrm{sr}}$ scales as $E_{\mathrm{sr}} \approx P_{\mathrm{sr}} \sigma_{\mathrm{sr}} \propto z^{3 / 2}[16-20]$.

Seeded FELs can operate either in the amplifier "direct seeding" scheme, or in the high gain harmonic generation (HGHG) configuration [21-24], where the seed in a first undulator (modulator) is used to induce an energy-density modulation in the electron beam longitudinal phase space. This bunched beam then emits a higher order harmonics $\lambda_{\text {rad }}=\lambda_{\text {seed }} / n$ in a following undulator (radiator). This scheme can be repeated in a multiple stage cascade of modulators and radiators, extending the operation 


\begin{tabular}{llllll}
$\lambda=400 \mathrm{~nm}$ & $\lambda=200 \mathrm{~nm}$ & $\lambda=200 \mathrm{~nm}$ & $\lambda=200 \mathrm{~nm}$ & $\lambda=200 \mathrm{~nm}$ & $\lambda=200 \mathrm{~nm}$ \\
\hline $1 \mathrm{UM}$ mod. & $1 \mathrm{UM} \mathrm{rad}$. & $2 \mathrm{UM} \mathrm{rad}$ & $3 \mathrm{UM} \mathrm{rad}$. & $4 \mathrm{UM} \mathrm{rad}$. & 5 UM rad.
\end{tabular}

FIG. 1 (color online). Undulator layout of the FEL cascade at SPARC. The gap of each undulator section can be set independently between 8 and $25 \mathrm{~mm}$, so that the undulators may be tuned at $\lambda_{\text {seed }}=400 \mathrm{~nm}$ (corresponding to $K=2.19$ ) as modulator, or at $\lambda_{\text {rad }}=200 \mathrm{~nm}(K=1.18)$ as radiator.

wavelength toward a range where seed sources are not available. In a FEL cascade, at the transition from a modulator to a radiator, the high frequency components in the leading edge of the pulse induce the growth of a new superradiant pulse at the harmonic frequency, which then grows, according to the above scaling relations, in the next undulator [18]. A cascaded superradiant configuration in principle permits to simultaneously decrease both the wavelength to the subnanometer scale and the pulse duration towards the femto- to attosecond frontier.

In this Letter we present the first demonstration of the generation of a superradiant pulse in the long radiator of a single stage cascaded FEL, by seeding the modulator with an external laser.

The experiment was performed at the UV seeded FEL of the SPARC facility [25]. The six SPARC undulators (75 periods each, period length $\lambda_{u}=2.8 \mathrm{~cm} \mathrm{[26])} \mathrm{were}$ configured to establish an HGHG FEL, with the first undulator tuned with the resonance at $400 \mathrm{~nm}$, playing the role of modulator, and the other five tuned at $200 \mathrm{~nm}$, as radiators (see Fig. 1).

The matching of the electron beam is ensured combining the natural undulator's vertical focusing with the horizontal focusing of quadrupoles placed in the undulator intersections. A periodic matching condition has been imposed on the beam Twiss parameters in the long radiators [27], with a constraint on the beam size in the first modulator. The resulting average Twiss $\beta$ functions in the radiator are $\left\langle\beta_{x}\right\rangle=\left\langle\beta_{y}\right\rangle \cong 2.75 \mathrm{~m}$ and $\left\langle\beta_{x}\right\rangle \cong 3 \mathrm{~m},\left\langle\beta_{y}\right\rangle \cong 2.45 \mathrm{~m}$ in the modulator. The FEL was seeded with the second harmonic $\left(\lambda_{\text {seed }}=400 \mathrm{~nm}\right)$ of a Ti:sapphire regenerative amplifier driven by the same oscillator of the photocatode laser system. A laser pulse energy of $E_{\text {seed }}=2 \mu \mathrm{J}$ (of duration $\sigma_{\text {seed }}=50 \mathrm{fs}$ ) was sufficient to seed the modulator

TABLE I. Main parameters of the electron beam. The beam energy uncertainty is related to the pulse to pulse jitter and does not respect the precision of the measurement.

\begin{tabular}{lcc}
\hline \hline Parameter & $(\mathrm{A})$ & $(\mathrm{B})$ \\
\hline$Q(\mathrm{pC})$ & $310 \pm 12$ & $260 \pm 10$ \\
$E(\mathrm{MeV})$ & $174.3 \pm 0.4$ & $173.2 \pm 0.4$ \\
$I(\mathrm{~A})$ & $54 \pm 5$ & $38.5 \pm 4$ \\
$\epsilon_{x}(\mathrm{~mm} \cdot \mathrm{mrad})$ & $2.6 \pm 0.2$ & $3.3 \pm 0.2$ \\
$\epsilon_{y}(\mathrm{~mm} \cdot \mathrm{mrad})$ & $2.4 \pm 0.2$ & $3.3 \pm 0.2$ \\
$(\Delta E / E)_{\text {proj }}$ & $(9.2 \pm 1) \times 10^{-4}$ & $(4.3 \pm 1) \times 10^{-4}$ \\
$(\Delta E / E)_{\text {slice }}$ & $(5.5 \pm 1) \times 10^{-4}$ & $(2.5 \pm 1) \times 10^{-4}$ \\
$\Delta \tau(p s)$ & $2.3 \pm 0.1$ & $2.4 \pm 0.1$ \\
\hline \hline
\end{tabular}

close to the onset of saturation. The saturation energy is roughly given by $E_{\text {sat }}=\sqrt{2 \pi} \sigma_{\text {seed }} \rho I E / e_{0} \approx 3.5 \mu \mathrm{J}$, with $e_{0}$ the electron charge, the Pierce parameter $\rho \sim 3 \times 10^{-3}$, and the other parameters defined in Table I (A).

Proof of superradiance may be obtained by verifying the expected energy or power scaling with the charge or beam current. Unfortunately, any variation of the peak current in a space charge dominated, high brightness accelerator, such as SPARC, can impact other beam parameters affecting the FEL process, such as the emittances and optics functions. Therefore we have verified the trend of the energy growth along the undulator mentioned above and compared these results with detailed numerical particle simulations using the FEL code GENESIS 1.3 [28], where the input parameters were varied according to the standard deviations in Table I. In addition, we observed the evidence of structures in the radiation spectrum measured along the radiator, and the emission of higher order harmonics at the end of the radiator, which is typical of a beam in saturated conditions.

The main output radiation diagnostic is an in-vacuum spectrometer [25] (normal incidence grating imaging the variable entrance slit on a UV grade CCD camera, Versarray, 1300B-Princeton Instruments), allowing simultaneous single shot measurements of the vertical and spectral distributions and of the absolute pulse energy [25].

The measured energy in the radiator as a function of the coordinate $z$ along the undulator is shown (black squares) in Fig. 2, for the conditions in column (B) of Table I. The

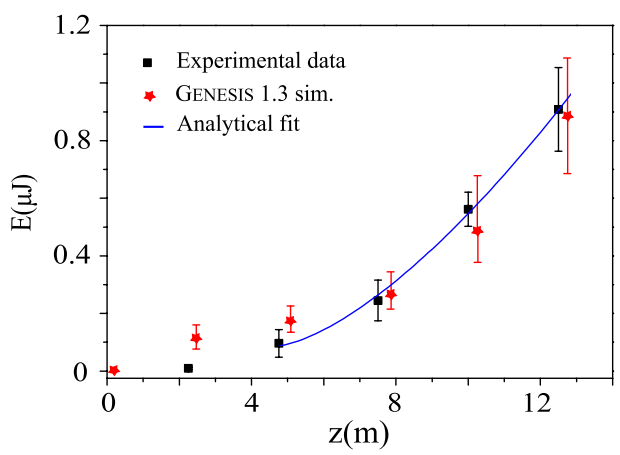

FIG. 2 (color online). Growth of the radiation energy at $\lambda_{\text {rad }}=200 \mathrm{~nm}$ along the radiator coordinate $z$. Black squares: experimental data. Red stars: simulation with GENESIS 1.3. Blue solid line: $a+b\left(z-z_{0}\right)^{3 / 2}$ analytical fit, with $a=0.096 \mu \mathrm{J}$, $b=3.9 \times 10^{-2} \mu \mathrm{J} / \mathrm{m}^{1.5}$ and $z_{0}=5.12 \mathrm{~m}$. Electron beam parameters of Table I (B). Data averaged over 100 shots. Error bars represent one standard deviation. 
results of simulations made with GENESIS 1.3 with input parameters from Table I (B) [current $I=(38.5 \pm 4) A$, emittance $\epsilon_{x-y}=(3.3 \pm 0.2) \mathrm{mmmrad}$, energy spread $\left.\Delta E / E=(2.8 \pm 1) \times 10^{-4}\right]$ are displayed as red stars. The simulated data were averaged over 100 random sets. Furthermore, a least squares fit of the energy growth vs $z$ at the end of the last four radiator modules exhibits a reasonable agreement with the scaling $E \propto z^{3 / 2}$ (see caption of Fig. 2).

The evolution of the spectrum along the radiator is shown in Fig. 3 for the beam parameters Table I, (B). The spectra are acquired by progressively closing the undulator gaps at the resonance condition, in order to enable the coupling between electrons and radiation only in the desired undulator longitudinal range. After closing

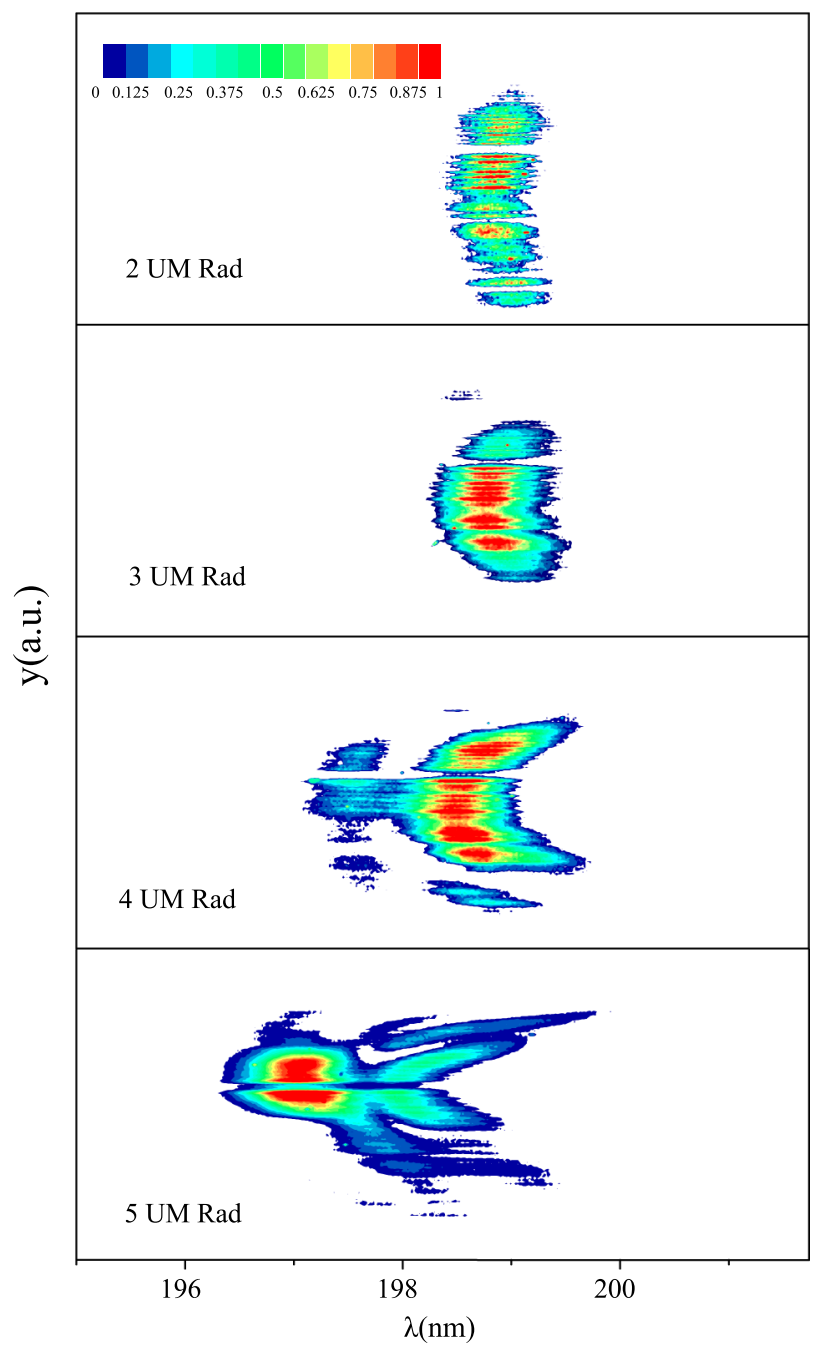

FIG. 3 (color online). Single shot spectra-spatial measurements along the radiator at $\lambda=200 \mathrm{~nm}$ for the beam of Table I (B). The vertical axis on the images of the spectra represents the vertical position on the input spectrometer slit. The images were taken after the third (second section of the radiator), the fourth, and the fifth section, and at the end of the undulator, respectively from the top to the bottom. the third radiator, sideband structures and an evident blueshift, appear in the spectrum. A qualitatively similar behavior is observed in the GENESIS 1.3 simulations.

Statistics of 100 consecutive spectral acquisitions reveal a positive correlation between energy and bandwidth along the radiator (Fig. 4). A substantial increase of the scatter of the data is observed after the third radiator. An averaged energy value of $\langle E\rangle=0.9 \pm 0.12 \mu \mathrm{J}$ at $\lambda_{\text {rad }}=200 \mathrm{~nm}$ is detected at the end of the undulator, with a peak value $E_{\text {peak }}=1.7 \mu \mathrm{J}$. Similar measurements done with the beam (A) were affected by energy fluctuations due to a phase jitter between the photoinjector laser and the accelerator radio frequency. Nevertheless, the higher beam quality led to a larger average energy per pulse $\langle E\rangle=1.6 \pm 2.3 \mu \mathrm{J}$, with a considerable dispersion due to fluctuations. Several shots exceeding $5 \mu \mathrm{J}$ were observed, with a maximum recorded value of $18 \mu \mathrm{J}$, about an order of magnitude larger than the saturation threshold in the radiator $\left(E_{\mathrm{sat}} \sim 2 \mu \mathrm{J}\right)$.

Another signature of saturation is given by the emission of higher order harmonics. The spectral data, averaged over 100 shots, are shown for harmonics up to the fifth in Fig. 5 [beam conditions Table I, (B)]. Higher order harmonics were out of the spectrometer spectral range of operation and could not be measured. The energy per pulse at each harmonic wavelength was reconstructed from the recorded data and is shown in Fig. 6. In comparison, a similar acquisition in beam conditions (A) led to an energy per pulse retrieved from the third harmonic spectrum, averaged over a set of 30 shots, of $\langle E\rangle=39.9 \pm 23.8 \mathrm{~nJ}$.

We studied for the first time the evolution at the onset of saturation, of a HGHG FEL in the transition from the modulator to the radiator. The modulator has been seeded by a short pulse of intensity comparable to the modulator saturation intensity, in order to induce superradiance. A superradiant pulse is expected to pass through the stage of a cascade FEL and to regenerate itself at the wavelength of the radiator. We have observed the evolution of the

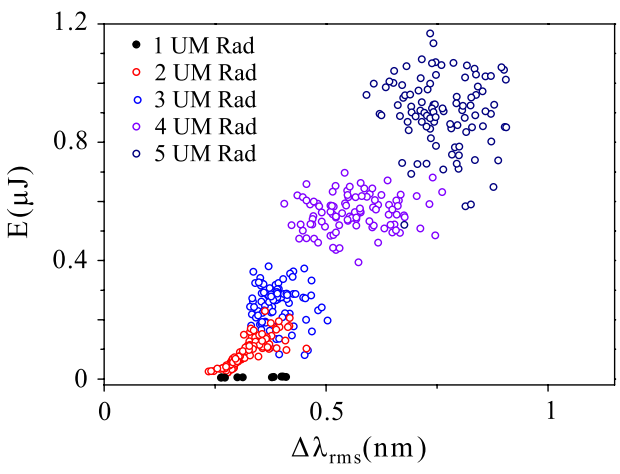

FIG. 4 (color online). Radiation energy $E(\mu \mathrm{J})$ vs bandwidth $\Delta \lambda_{\text {rms }}(\mathrm{nm})$ at different position along the undulator. The statistics of 100 consecutive single shots for beam B is shown, except after 1 UM Rad (black dots) where averages of 10 shots over $1 \mathrm{~s}$ are plotted. 

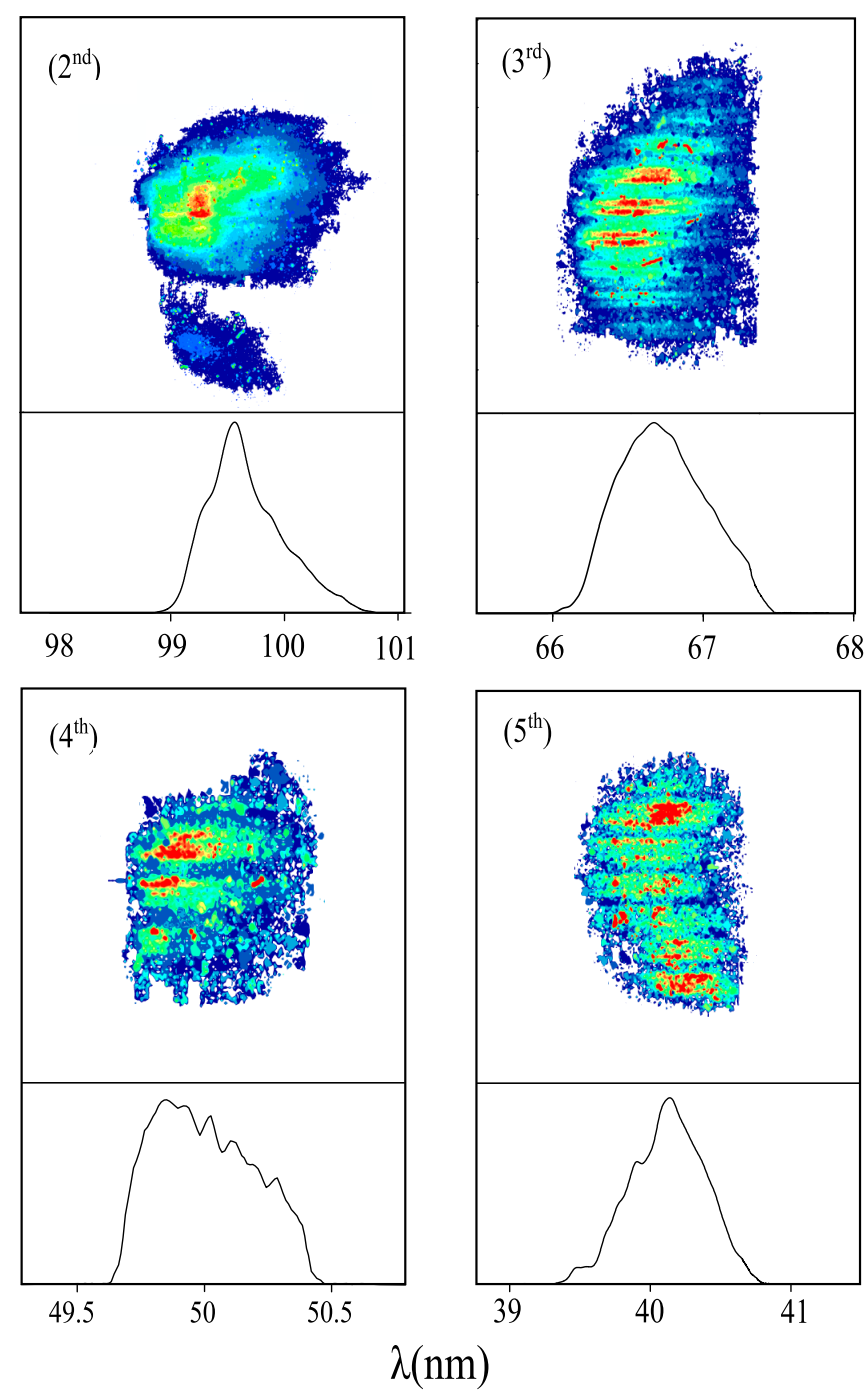

FIG. 5 (color online). Second, third, fourth, and fifth harmonics of the radiator for beam (B). Spectra-spatial measurements at the end of the radiator obtained averaging over a time of $20 \mathrm{~s}$ (30 $\mathrm{s}$ for the 5th harmonics). In the lower boxes, intensity of the radiation spectrum vs wavelength. Same color palette as Fig. 3. The third to fifth spectra were acquired by interposing an aluminum (Al) filter to reduce the background noise due to lower harmonics at the zero order of the grating. The horizontal lines are due to the presence of a mesh sustaining the $\mathrm{Al}$ layer.

pulse in the radiator by measuring its energy and spectral properties. Energy per pulse substantially larger than the FEL saturation energy, without applying any undulator taper, has been observed. An energy scaling vs the longitudinal position along the undulator of the type $E \propto z^{3 / 2}$ has been measured, in satisfactory agreement with simulations. Sidebands in the spectrum appear after three sections of the radiator and the emission of higher order harmonics as expected in superradiance [29] has been confirmed.

The feasibility of a superradiant cascaded FEL configuration has been proved, providing insight into novel methods for producing short and high frequency pulses.

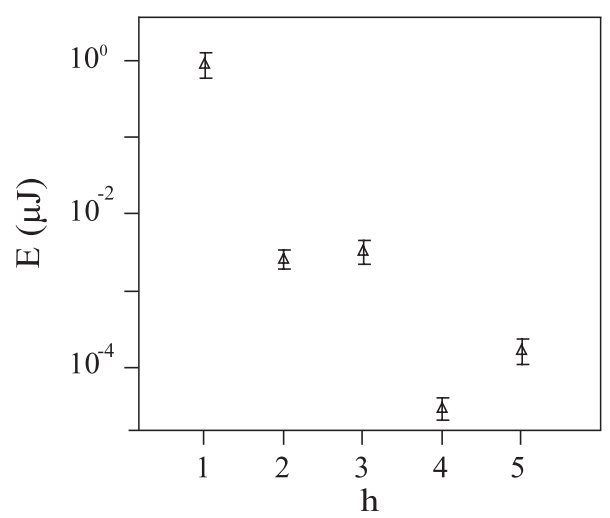

FIG. 6. Energy at the fundamental and higher order harmonics in the radiator. The energy of the second harmonics is $E=2.6 \pm 0.7 \mathrm{~nJ}$, of the third: $E=3.3 \pm 1.1 \mathrm{~nJ}$, of the fourth: $E=0.03 \pm 0.01 \mathrm{~nJ}$, and of the fifth: $E=0.17 \pm 0.06 \mathrm{~nJ}$.

We wish to acknowledge the entire SPARC team for the long hours spent in the control room, making this work possible. This work has been partially supported by Ministero dell''Istruzione, dell"Università e della Ricerca (DM1834 RIC.4-12-2002) and by the EU sixth framework program, Contract No. 011935 EUROFEL.

*Luca.Giannessi@enea.it

[1] O. Gessner et al., Science 311, 219 (2006).

[2] F. Tavella, N. Stojanovic, G. Geloni, and M. Gensch, Nat. Photonics 5, 162 (2011).

[3] K. J. Gaffney and H. N. Chapman, Science 316, 1444 (2007).

[4] Q. Lin, J. Li, and G. C. Guo, J. Phys. B 39, 3649 (2006).

[5] C. M. Guenther et al., Nat. Photonics 5, 99 (2011).

[6] M. F. Kling and M. J. J. Vrakking, Annu. Rev. Phys. Chem. 59, 463 (2008).

[7] P. Emma et al., Nat. Photonics 4, 641 (2010).

[8] T. Ishikawa et al., Nat. Photonics 6, 540 (2012).

[9] K.-J. Kim, Phys. Rev. Lett. 57, 1871 (1986).

[10] J.R. Pierce, Traveling-Wave Tubes (van Nostrand Co., New York, 1950).

[11] R. Bonifacio, C. Pellegrini, and L. M. Narducci, Opt. Commun. 50, 373 (1984).

[12] R. Bonifacio, L. De Salvo, P. Pierini, N. Piovella, and C. Pellegrini, Phys. Rev. Lett. 73, 70 (1994).

[13] L. Giannessi et al., Phys. Rev. Lett. 106, 144801 (2011).

[14] G. Marcus et al., Appl. Phys. Lett. 101, 134102 (2012).

[15] R. H. Dicke, Phys. Rev. 93, 99 (1954).

[16] T. Watanabe, X. J. Wang, J. B. Murphy, J. Rose, Y. Shen, T. Tsang, L. Giannessi, P. Musumeci, and S. Reiche, Phys. Rev. Lett. 98, 034802 (2007).

[17] R. Bonifacio and F. Casagrande, Nucl. Instrum. Methods Phys. Res., Sect. A 239, 36 (1985).

[18] L. Giannessi, P. Musumeci, and S. Spampinati, J. Appl. Phys. 98, 043110 (2005).

[19] R. Bonifacio, L. De Salvo Souza, P. Pierini, and N. Piovella, Nucl. Instrum. Methods Phys. Res., Sect. A 296, 358 (1990). 
[20] R. Bonifacio, C. Maroli, and N. Piovella, Opt. Commun. 68, 369 (1988).

[21] L. H. Yu, Phys. Rev. A 44, 5178 (1991).

[22] L. H. Yu et al., Science 289, 932 (2000).

[23] A. Doyuran et al., Phys. Rev. Lett. 86, 5902 (2001).

[24] M. Labat et al., Phys. Rev. Lett. 107, 224801 (2011).

[25] L. Giannessi, Phys. Rev. ST Accel. Beams 14, 060712 (2011).
[26] M. Quattromini et al., in Proceedings of the 2008 EPAC Conference (www.jacow.org, 2008), p. WEPC124.

[27] M. Quattromini, M. Artioli, E. Di Palma, A. Petralia, and L. Giannessi, Phys. Rev. ST Accel. Beams 15, 080704 (2012).

[28] S. Reiche, Nucl. Instrum. Methods Phys. Res., Sect. A 429, 243 (1999).

[29] L. Giannessi et al., Phys. Rev. Lett. 108, 164801 (2012). 